\title{
Hypersonic Flow over a Flat Plate: CFD Comparison with Experiment
}

\author{
Andrew J. Lofthouse* \\ U.S. Air Force Institute of Technology, Wright-Patterson AFB, OH, 45433 \\ Iain D. Boyd ${ }^{\dagger}$ \\ The University of Michigan, Ann Arbor, MI, 48109
}

\begin{abstract}
Experimental results of a hypersonic flow over a flat plate are compared with a CFD simulation. Slip boundary conditions are employed in the CFD simulation, with the accommodation coefficient being varied between 0.5 and 1.0. Detailed velocity profiles in both the $x$ - and $y$-directions are compared with the experimental data, as well as slip velocity at the wall. A brief comparison is also made to similar simulation results using DSMC.
\end{abstract}

\section{Introduction}

The design of hypersonic vehicles requires accurate prediction of the gas flow around the vehicles surfaces. During it's trajectory through an atmosphere, a hypersonic vehicle will experience vastly different flow regimes due to the variation of atmospheric density with altitude. In addition, the high temperatures encountered due to the high velocities cause dissociation and ionization of the atmospheric gases. Reproduction of these varied flow conditions in ground-based laboratory facilities is both expensive and technically challenging. Hence, there is an extremely important role for computational models in the development of hypersonic vehicles.

In the continuum regime flows around hypersonic vehicles can be accurately simulated using traditional Computational Fluid Dynamics (CFD) by solving either the Euler or preferably the Navier-Stokes (NS) equations. These continuum methods assume small perturbations from local thermodynamic equilibrium. In the rarefied flow regime the flow can be computed using the direct simulation Monte Carlo (DSMC) method. ${ }^{1}$ The DSMC method does not depend on assumptions involving a small perturbation from equilibrium and hence is more accurate than CFD methods for non-equilibrium flows. Generally speaking, CFD methods for solving the NS equations are about an order of magnitude faster than the DSMC method. Note that in continuum regimes, locally a flow may behave like a rarefied flow if the local characteristic length scale is very small.

Previous work by the authors comparing CFD and DSMC focused purely on numerical results, with CFD simulations being compared directly to DSMC simulations. ${ }^{7-10}$ In particular, the walls were assumed to be fully diffusive; that is, the gas molecules colliding with the wall were assumed to accommodate fully to the wall conditions. Hence, an accommodation coefficient of unity was used for the CFD slip boundary conditions. In this paper, two-dimensional CFD solutions are compared with experimental measurements of a hypersonic flow of nitrogen over a flat plate. ${ }^{4}$ Several different values for the accommodation coefficient are evaluated. In addition, the CFD solutions are also indirectly compared to DSMC solutions of the same flow. ${ }^{11}$ Thus, the relative accuracy of CFD and DSMC can be evaluated against a realistic flow.

\section{Background and Experimental Results}

The experimental results of a hypersonic flow over a flat plate are taken from Cecil and McDaniel. ${ }^{4}$ Measurements of the flow were taken using planar laser-induced flourescence (PLIF). A hypersonic flow of nitrogen,

\footnotetext{
*Assistant Professor, Department of Aeronautics and Astronautics, 2950 Hobson Way; Andrew.Lofthouse@afit.edu. Senior Member AIAA.

†Professor, Department of Aerospace Engineering, 1320 Beal Avenue; iainboyd@umich.edu. Associate Fellow AIAA.

"The views expressed in this paper are those of the author and do not reflect the official policy or position of the United States Air Force, Department of Defense, or the U.S. Government"
} 
with a Mach number of approximately 11.9 at the leading edge of the flat plate, was created by expanding an iodine-seeded flow of nitrogen from a settling chamber through a thin, circular orifice and over the model in a continuously evacuated vacuum chamber. A PLIF image of the flow is seen in Figure 1. Here, the gas is seen expanding in a free jet from an orifice at the left of the image to the right over the model. The process of expanding the flow through the orifice created a barrel shock (the top portion of which is clearly seen at the top of the figure) terminated by a normal shock (not shown). The expanding flow from the orifice within the barrel shock, where the model was located, was not uniform, but instead varied radially. On the left of the figure, the light-colored rays extending from the orifice upstream of the model shows the radial variation of the velocity. The shock formed by the flow over the model is also clearly shown.

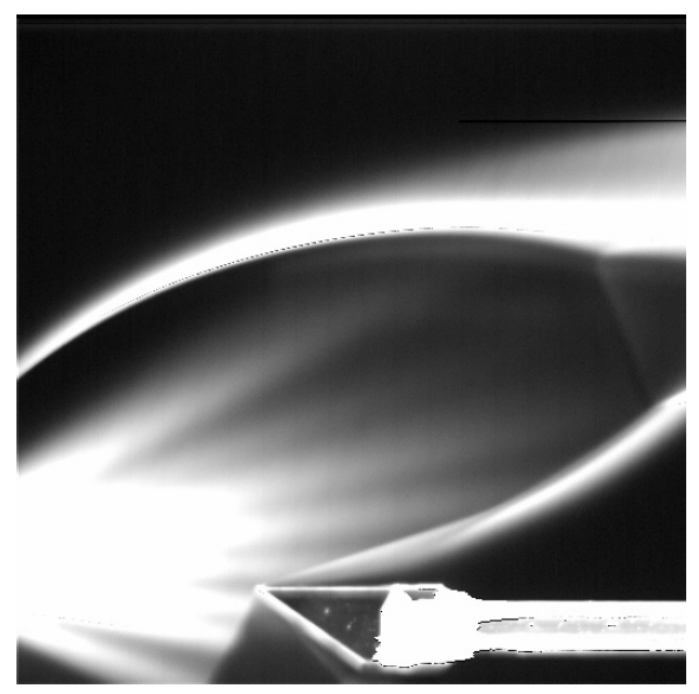

Figure 1. PLIF image of hypersonic flow over the flat plate model (from Ref. ${ }^{4}$ ). The gas expands in a free jet from the left, creating a barrel shock (near the top of the figure). Note the light-colored rays extending from the orifice upstream, showing the radial variation of the velocity. Note also the shock attached to the plate leading edge.

The temperature of the settling chamber was approximately $300 \mathrm{~K}$. Expansion through the orifice reduced the temperature to about $11.5 \mathrm{~K}$ near the leading edge of the model. This temperature is sufficiently low that no vibrational activation is expected.

The density at the leading edge was about $3 \times 10^{-4} \mathrm{~kg} / \mathrm{m}^{3}$, giving a global Knudsen number (based on a hard-sphere mean free path and the flat plate length of $20 \mathrm{~mm}$ ) of about 0.009 . This is comparable to the global Knudsen number value of 0.01 for the cases discussed in previous work. ${ }^{7}$ In those cases, CFD gave fairly accurate results for a hypersonic flow over a wedge, with the exception of the region near the leading edge where nonequilibrium effects were significant.

Velocity magnitude contours and streamlines from the experimental results are shown in Figure 2. Note that the length coordinates, $x$ and $y$, are nondimensionalized by the diameter, $d=0.5 \mathrm{~mm}$, of a small nozzle built into the plate at $x / d=30$, or, equivalently, $x=15 \mathrm{~mm}$. In further experiments, a jet was issued from the nozzle to simulate a reaction control system. The flat plate leading edge is located at $x / d=0$, and the trailing edge is located at $x / d=40$. The streamlines in the freestream again illustrate the nonuniform flow due to the source-like nature of the flow expanding from the orifice.

It should also be noted that there is a pocket of very low velocity gas near the trailing edge of the model (between $x / d=40$ and 50). There is evidence of an adverse pressure gradient at this location that will be discussed in further detail below.

Experimental data for the time-averaged velocity was provided for $y / d=0$ to 15 at several locations along the plate from $x / d=0$ to 40 in increments of 5. This corresponds to $y=0$ to $7.5 \mathrm{~mm}$ and $x=0$ to $20 \mathrm{~mm}$ in increments of $2.5 \mathrm{~mm}$. The uncertainties of the $U$ and $V$-velocity components were estimated to be on the order of $50 \mathrm{~m} / \mathrm{s}$ and $30 \mathrm{~m} / \mathrm{s}$, respectively. ${ }^{4}$ 


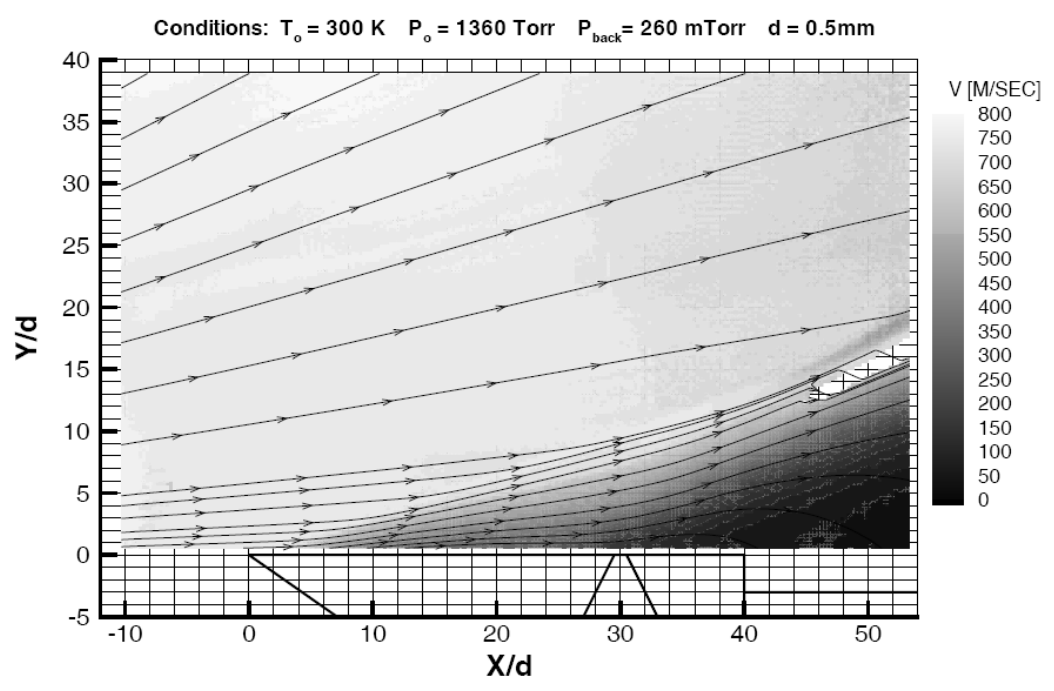

Figure 2. Measured velocity contours and streamlines on the centerplane of the hypersonic flow over a flat plate (from Ref. ${ }^{4}$ ). A small nozzle is built into the plate at $x / d=30$. Note the radial variation in velocity upstream of the flat plate, and the pocket of low velocity gas near the trailing edge at $x / d=40$.

\section{Computational Results: DSMC}

Padilla and Boyd ${ }^{11}$ conducted DSMC simulations of this flow using pure nitrogen and the variable soft-sphere (VSS) DSMC model. ${ }^{1}$ The nonuniform inflow velocity profiles used for the DSMC case were taken from the experimental data. The temperature and the particle number density were calculated assuming an isentropic expansion from the settling chamber conditions of $T=300 \mathrm{~K}$ and $p=1.79 \mathrm{~atm}$. These inflow boundary conditions are shown in Figure 3, where the streamwise, or $x$-direction, velocity component is $U$ and the wall-normal, or $y$-direction, velocity component is $V$. Note that the particle number density has been converted to the mass density. The variation of the $V$-velocity is particularly important; while the $U$-velocity varies by only $6 \mathrm{~m} / \mathrm{s}$ across the inflow boundary, the $V$-velocity varies by nearly $150 \mathrm{~m} / \mathrm{s}$ (from about $20 \mathrm{~m} / \mathrm{s}$ at $y=0 \mathrm{~mm}$ to about $160 \mathrm{~m} / \mathrm{s}$ at $y=7.5 \mathrm{~mm}$. The inflow boundary was set $2 \mathrm{~mm}$ upstream of the flat plate leading edge. A wall temperature of $300 \mathrm{~K}$ was assumed.

DSMC simulations were computed with two different gas-surface interaction models and tangential momentum accommodation coefficients of 0 to 1.0. The DSMC simulations show that a tangential momentum accommodation coefficient of 1.0 gives the best agreement with the measured data for the streamwise-component of velocity $(U)$, while a tangential momentum accommodation coefficient of 0.75 gives the best overall agreement with the wall-normal component of velocity $(V)$. An average accommodation coefficient of 0.875 gave good overall agreement with the experimental data.

\section{Computational Results: CFD}

CFD solutions are obtained using the Gökçen ${ }^{5}$ slip boundary conditions, as described earlier. ${ }^{10}$ Simulations performed using the Maxwell and Lockerby's wall-function ${ }^{6}$ slip boundary conditions show very similar results, with the exception that the Gökçen slip conditions tend to show a larger slip velocity. This is not unexpected; previous simulations for similar flow conditions showed that the velocities predicted by all three slip conditions are very similar away from the wall, with the Gökçen simulations predicting the highest slip velocity. ${ }^{10}$ The higher slip velocity values predicted by the Gökçen slip conditions agree best with DSMC and the experimental data, for these flow conditions, as will be shown below.

Previous CFD results were compared with DSMC simulations using a variable-hard sphere (VHS) model. ${ }^{1}$ Thus, the viscosity in the CFD simulations are explicitly defined to be consistent with the VHS model. ${ }^{12}$ The present results also use the VHS viscosity model, with the values for nitrogen given in Table 1. Although the DSMC simulations used the VSS model, there are no significant differences expected at the low temperatures 


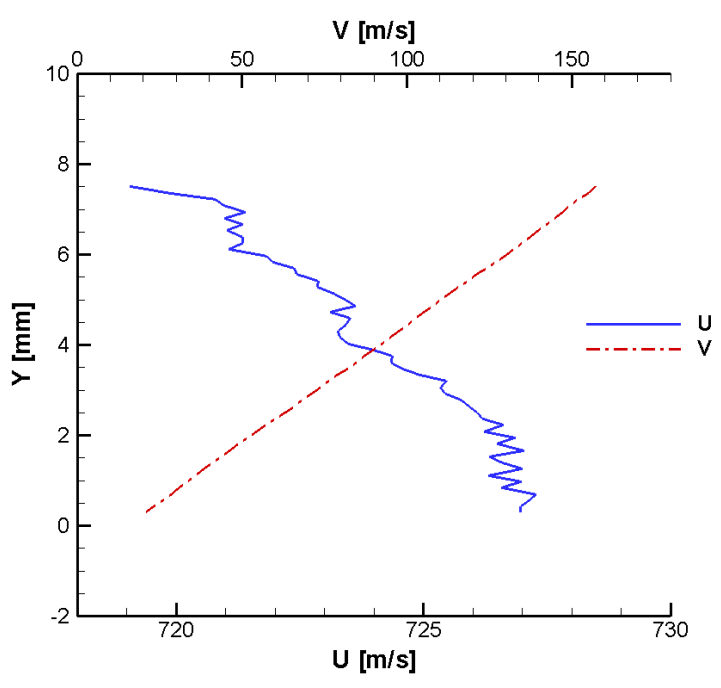

(a) Velocity components

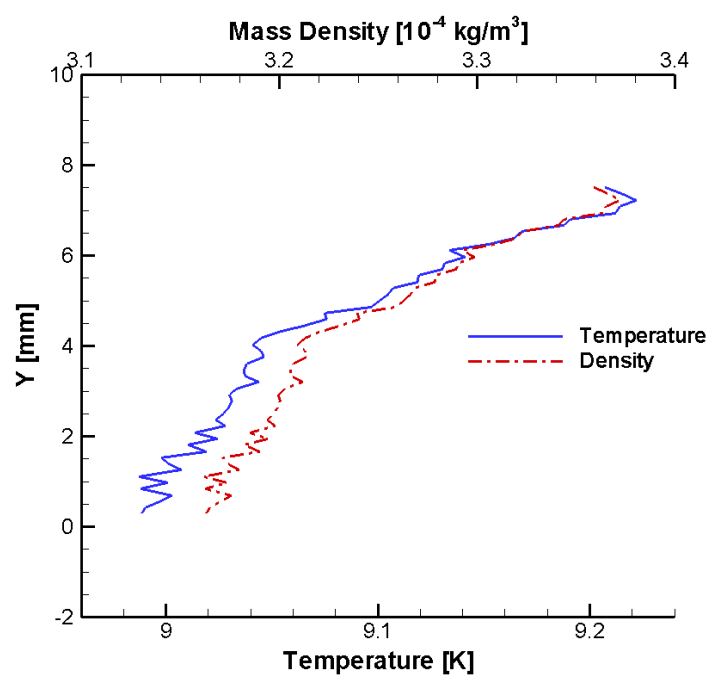

(b) Temperature and mass density

Figure 3. Inflow boundary conditions for a hypersonic flow over a flat plate. The inflow boundary conditions for the two-dimensional simulations vary with vertical distance from the plate $(y)$ to match the radial variation of the experimental conditions.

encountered. In addition, a test simulation using Blottner's curve fit for the viscosity ${ }^{2}$ yielded no discernable difference in the velocity profiles.

Table 1. Variable hard sphere (VHS) model parameters for nitrogen used in the computational simulations.

\begin{tabular}{|c|c|c|c|}
\hline Species & $\omega$ & $T_{\text {ref }}[\mathrm{K}]$ & $d_{\text {ref }}[\mathrm{m}]$ \\
\hline $\mathrm{N}_{2}$ & 0.7 & 290 & $4.110 \times 10^{-10}$ \\
\hline
\end{tabular}

The areas of the flow where the continuum hypothesis breaks down (or equivalently, where the flow is no longer in local thermodynamic equilibrium), can be quantified by the use of a continuum breakdown parameter. The breakdown parameter that appears to be most appropriate for hypersonic compressible flows, and that which is used here, is the gradient-length local (GLL) Knudsen number ${ }^{3,13}$

$$
\mathrm{Kn}_{\mathrm{GLL}}=\frac{\lambda}{Q}\left|\frac{d Q}{d l}\right|
$$

where the derivative is taken in the direction of the maximum gradient, and $Q$ is some quantity of interest such as density, pressure, temperature or velocity magnitude. When calculating $\mathrm{Kn}_{\mathrm{GLL}}$ based on velocity magnitude, the gradient is normalized by the maximum of the local velocity magnitude and the local speed of sound. It is generally assumed that continuum breakdown occurs whenever $\mathrm{Kn}_{\mathrm{GLL}}$ (based on the CFD solution) is greater than 0.05 .

The method used here for producing mesh-independent results is somewhat different than that used previously. Earlier studies were mainly concerned with obtaining correct values for the surface properties. Therefore, the surface property values were used to determine when a mesh-independent solution was obtained. It was found that mesh-independent solutions were most sensitive to the node spacing near the wall (for both the wedge and the cylinder cases) and in the streamwise direction (for the wedge case). The objective here, however, is to compare the flow field properties (specifically the velocity components) with the experimental measurements. Therefore, a mesh-independent solution is defined as one for which successively refined meshes produce no discernable differences in the velocity profiles. For this flat plate flow, the flow field properties (especially the $V$ component of velocity) is very sensitive to the node spacing in the wall-normal direction in the entire flow field, rather than simply near the wall. There is also some sensitivity to the node spacing in the streamwise direction, especially near the leading edge where the gradients are largest. 
In a similar manner to the DSMC simulations, here the accommodation coefficient is varied from 0.5 to 1.0. The computational domain consists of a rectangular area from $y=0$ to $20 \mathrm{~mm}$ and from $x=-2$ to $30 \mathrm{~mm}$.

\section{A. Flow Field}

The contours for the value of a continuum breakdown parameter, $\mathrm{Kn}_{\mathrm{GLL}},{ }^{8}$ as computed from the CFD solutions for two different accommodation coefficient values $(\sigma=1.0$ and 0.5$)$, are shown in Figure 4, and the density contours are shown in Figure 5. It is apparent that the flow is dominated by viscosity effects; in other words, the flow has a large amount of nonequilibrium present, since the transfer of momentum due to velocity gradients is an inherent nonequilibrium process. The largest values of the breakdown parameter are found in the shock region, and, to a lesser extent, the wake. It is interesting to note that the value of the breakdown parameter exceeds the critical value of 0.05 in most of the flow region behind the shock, due to the merging of the shock and the boundary layer. Nevertheless, the CFD solutions do give good agreement with the experimental data in these areas, as shown below.

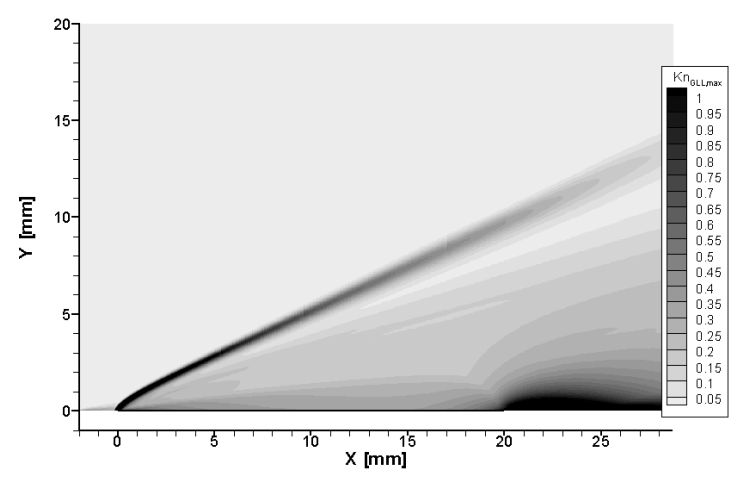

(a) $\sigma=1.0$

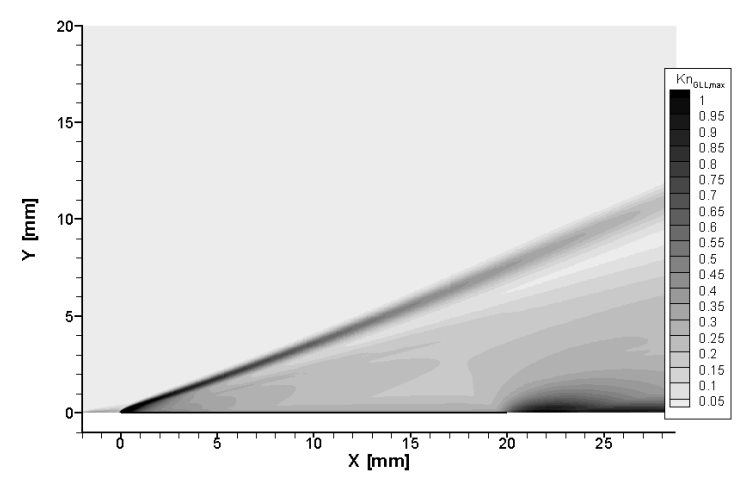

(b) $\sigma=0.5$

Figure 4. $\mathrm{Kn}_{\mathrm{GLL}}$ field for hypersonic flow over a flat plate. Significant nonequilibrium effects are expected at the leading edge and in the wake. Full accommodation increases the viscous effects, increasing the shock strength and the shock-boundary layer interaction that leads to a curved shock at the leading edge.

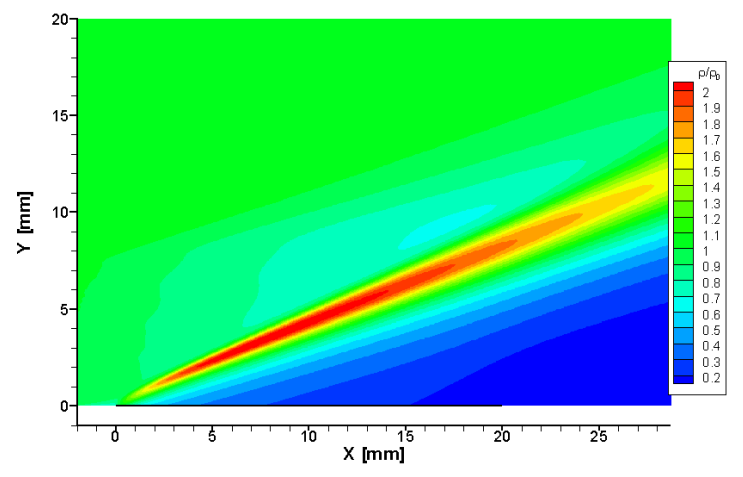

(a) $\sigma=1.0$

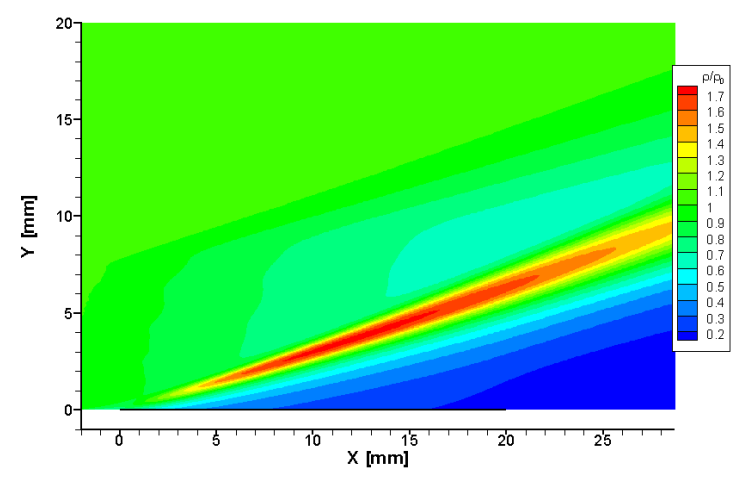

(b) $\sigma=0.5$

Figure 5. Density field for hypersonic flow over a flat plate. Full accommodation increases the viscous effects, increasing the compression across the shock, as well as the shock-boundary layer interaction that leads to a curved shock at the leading edge.

Reducing surface accommodation from full ( $\sigma=1.0$ ) to half ( $\sigma=0.5$ ) effectively reduces the effect of viscosity on the flow field. For full accommodation the shock is stronger, and the compression ratio is higher (with maximum density ratio of about 2.0 compared to 1.7 for half accommodation). In addition, the strong viscous-shock interaction at the leading edge is more apparent for full accommodation, as can be seen by the 
curvature of the shock in that area. Cecil and McDaniel ${ }^{4}$ mention that the shock wave angle of $18^{\circ}$ far exceeds the value of $4.8^{\circ}$ predicted by inviscid theory and that an effective angular displacement of $13-14^{\circ}$ by the boundary layer is necessary to produce the $18^{\circ}$ angle. The reduction in shock angle as the accommodation coefficient is reduced also demonstrates the reduced effect of viscosity as surface accommodation is reduced.

One can compare the velocity magnitude contour plots with streamlines from the CFD results in Figure 6 with the similar plot of the experimental data in Figure 2. First, note that the nonuniform inflow conditions appear to match the experimental results quite well. Also note that the surface accommodation in the experiment appears to be lower than 1.0, as the streamlines near the leading edge in the experimental results are not displaced upward as much as is shown in the numerical results with full accommodation. Finally, note that the computational results do not show the pocket of low velocity at the trailing edge that is apparent in the experimental results. (Recall that $x / d=40$ is the location of the trailing edge, and is equivalent to $x=20 \mathrm{~mm}$.) It appears that whatever causes the low velocity at the trailing edge (perhaps a region of high pressure) also causes the streamlines to be displaced upwards much more in the experimental results than is shown in the numerical results.

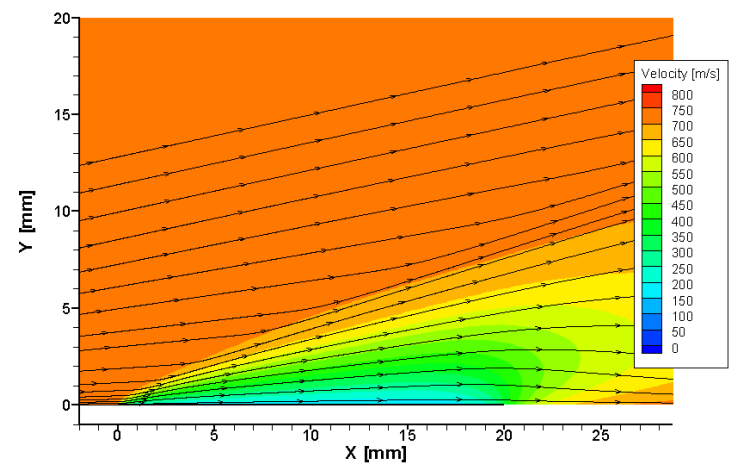

(a) $\sigma=1.0$

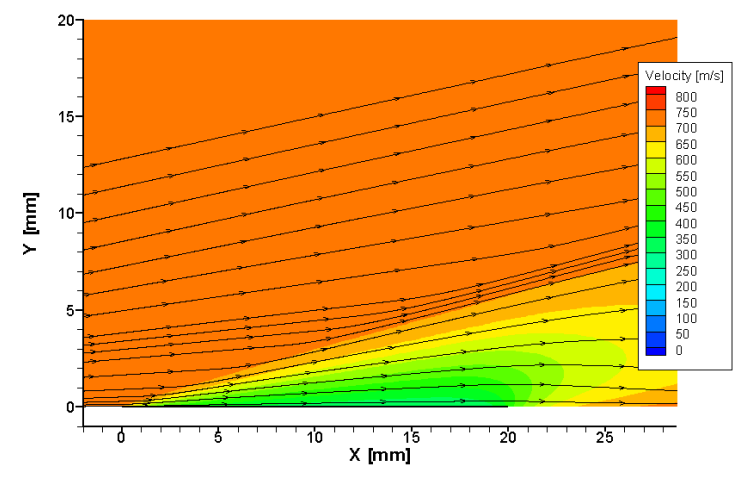

(b) $\sigma=0.5$

Figure 6. Computed velocity magnitude contours and streamlines for hypersonic flow over a flat plate. The half-accommodation results compare more favorably with the experimental results in Figure 2.

\section{B. Velocity Comparisons}

The flow velocity component results from each numerical simulation are compared with the experimental results at several locations along the flat plate. The $U$-velocity profiles are compared in Figure 7 and the $V$-velocity profiles are compared in Figure 8. The $U$ profiles show typical boundary layer behavior, including the non-zero velocity slip at the wall due to the rarefied nature of the flow. The $V$ profiles include the effects due to the shock.

The first profiles, at $x=0 \mathrm{~mm}$, show good agreement between the experiment and the simulations. Although there is slightly worse agreement in the $V$-velocity profile as $y$ increases, the numerical results remain well within the estimated level of uncertainty in the experimental data. The disagreement as $y$ increases is most likely due to incomplete inflow boundary condition modeling. The values used were derived from the experimental data, which is limited to values below $y=7.5 \mathrm{~mm}$. Although it can be assumed, by looking at the experimental streamlines in Figure 2, that the radial component of velocity continues to increase as $y$ increases, the boundary conditions implemented do not assume an increasing value of $V$ as $y$ increases beyond $y=7.5 \mathrm{~mm}$ (see Figure 8(a)). Nevertheless, the boundary conditions used in the simulations are assumed to be adequately correct.

Very near the leading edge, at $x=1.5 \mathrm{~mm}$, the flow solution with $\sigma=0.5$ shows the best agreement with the experiment, for both $U$ and $V$. Further down the plate, however, up to $x=12.5 \mathrm{~mm}$, flow solutions with more surface accommodation, $\sigma=60$ or 70 , agree best for $U$, while the solutions with $\sigma=50$ continue to agree best for $V$. Starting at $x=15 \mathrm{~mm}$, the agreement for $U$ progressively worsens, and the experimental $U$ profiles at $x=17.5$ and $20 \mathrm{~mm}$ strongly imply the presence of an adverse pressure gradient. The specific cause of the pressure gradient is not present in the simulations, and it is not surprising to see disagreement in the flow velocity predictions near the trailing edge.

It is not surprising that the lower surface accommodation values agree better nearer to the leading edge; less surface accommodation reduces the affect of viscosity (and increases the velocity slip)—it was shown previously 


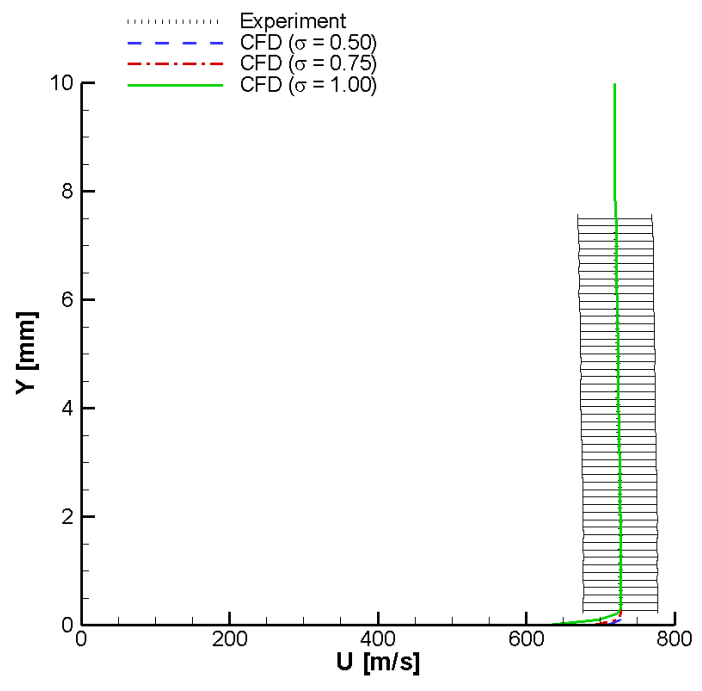

(a) $\mathrm{x}=0 \mathrm{~mm}$

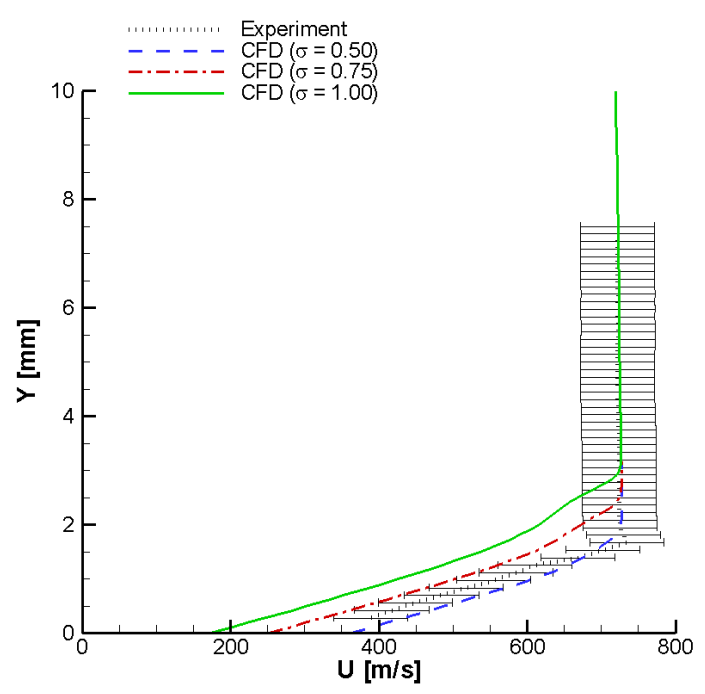

(c) $\mathrm{x}=5.0 \mathrm{~mm}$

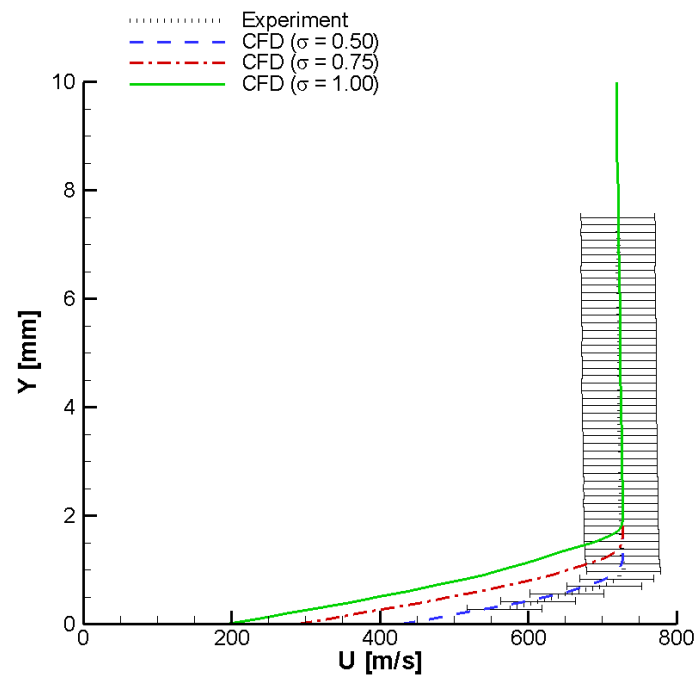

(b) $\mathrm{x}=2.5 \mathrm{~mm}$

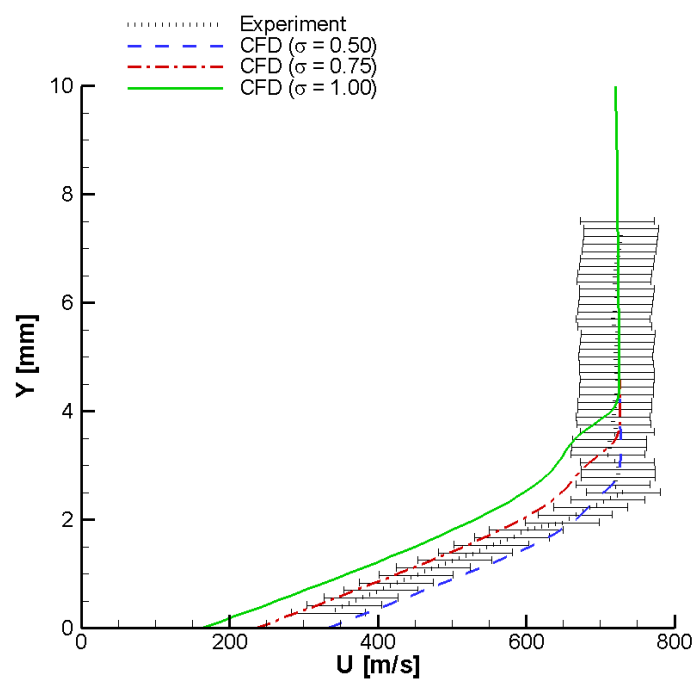

(d) $\mathrm{x}=7.5 \mathrm{~mm}$

Figure 7. Velocity component parallel to the surface (U) for a hypersonic flow over a flat plate. All CFD results match the experimental values at the leading edge, confirming that the inflow boundary conditions are correct. Simulations with higher values of surface accommodation match the experimental results best near the leading edge. 


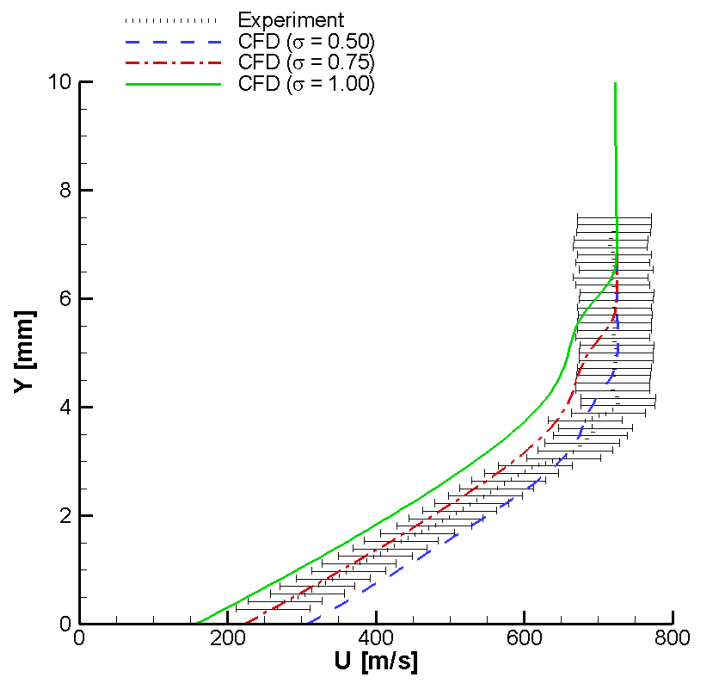

(e) $\mathrm{x}=12.5 \mathrm{~mm}$

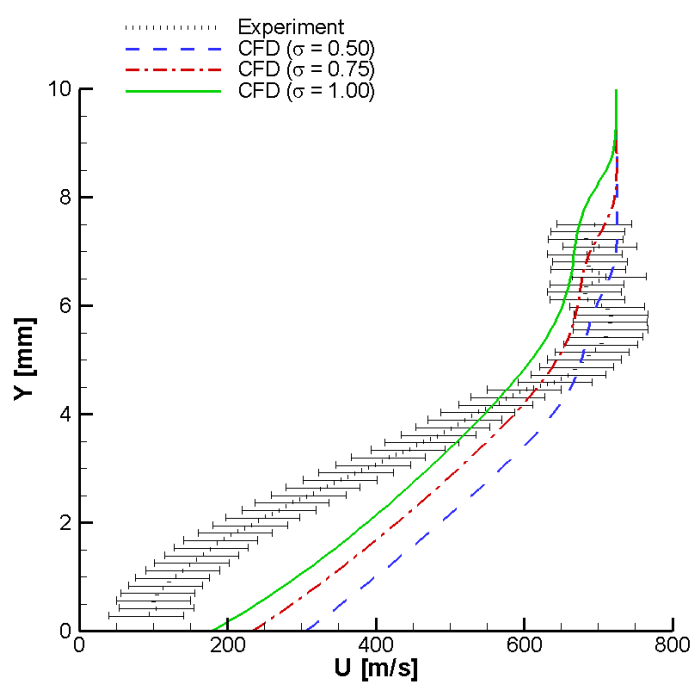

(g) $\mathrm{x}=17.5 \mathrm{~mm}$

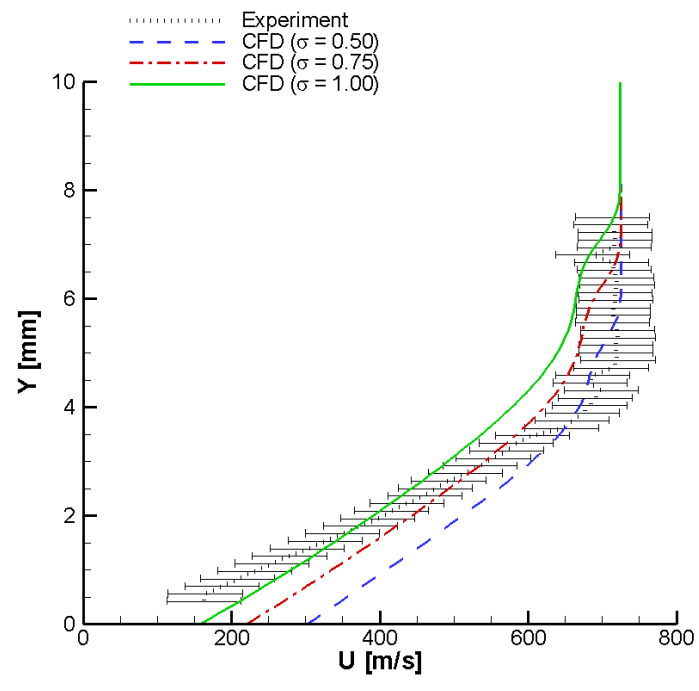

(f) $\mathrm{x}=15.0 \mathrm{~mm}$

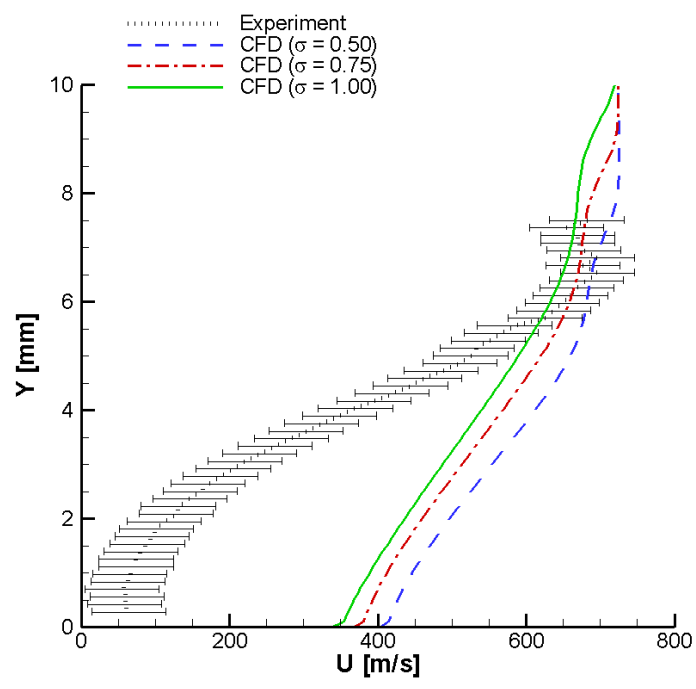

(h) $\mathrm{x}=20.0 \mathrm{~mm}$

Figure 7. Velocity component parallel to the surface (U) for a hypersonic flow over a flat plate (cont.). Downstream of the leading edge, CFD results with $\sigma=0.75$ agree best with experiment. The experimental velocity profiles near the trailing edge strongly suggest the presence of an adverse pressure gradient. 


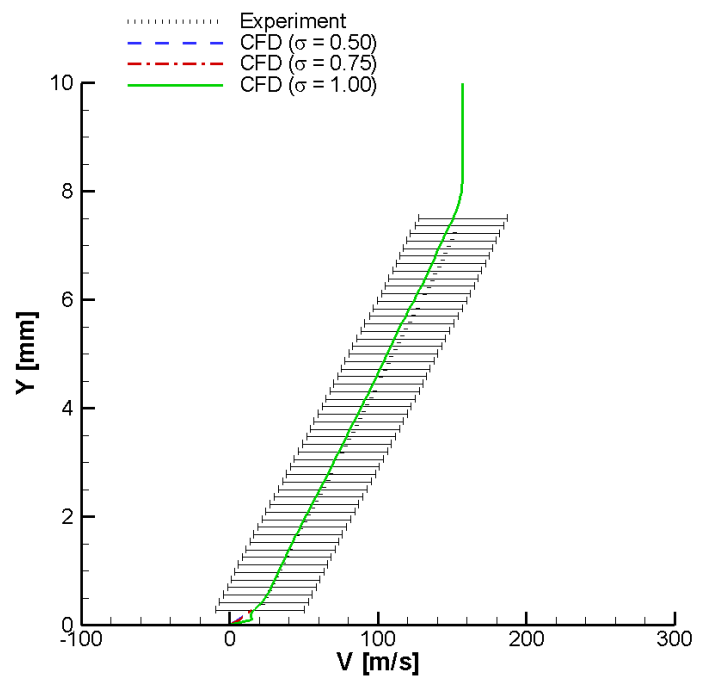

(a) $\mathrm{x}=0 \mathrm{~mm}$

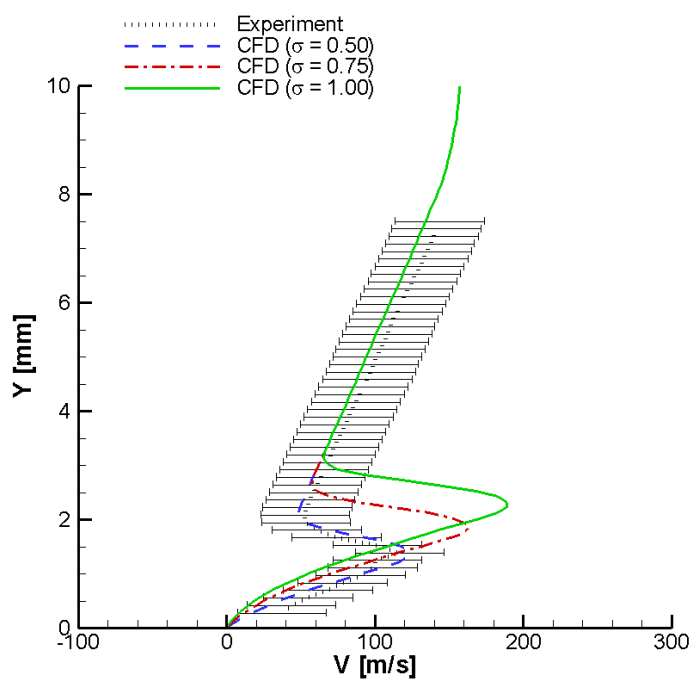

(c) $\mathrm{x}=5.0 \mathrm{~mm}$

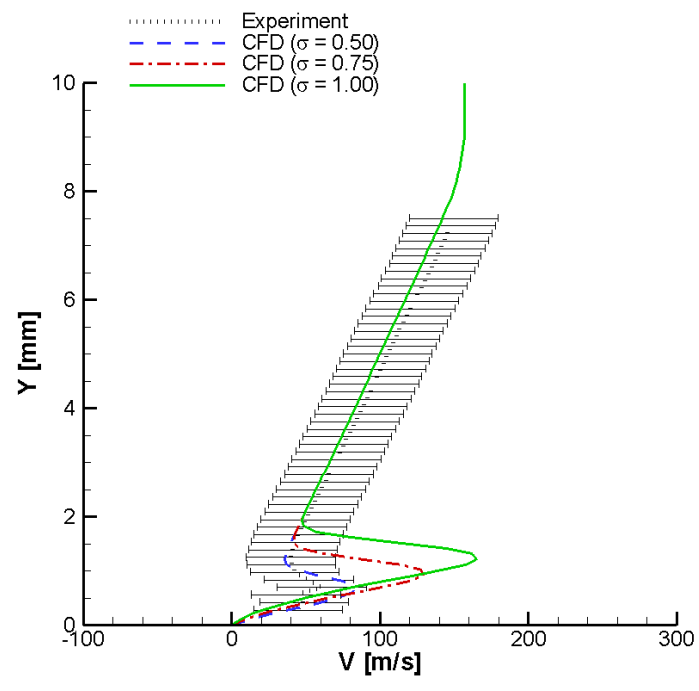

(b) $\mathrm{x}=2.5 \mathrm{~mm}$

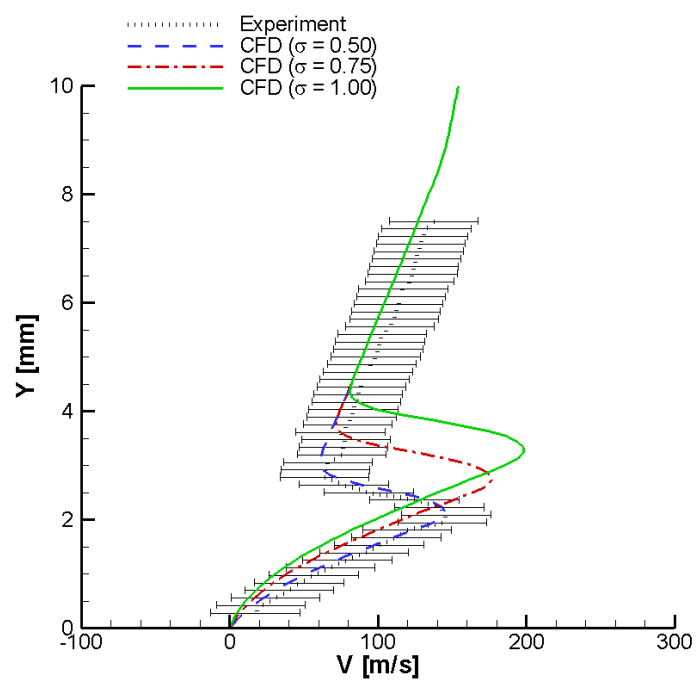

(d) $\mathrm{x}=7.5 \mathrm{~mm}$

Figure 8. Velocity component normal to the surface (V) for a hypersonic flow over a flat plate. The inflow boundary conditions match the experimental data where present (below $y=0.75 \mathrm{~mm}$ ). The computed velocity profiles through the shock match the experiment best for $\sigma=0.5$. 


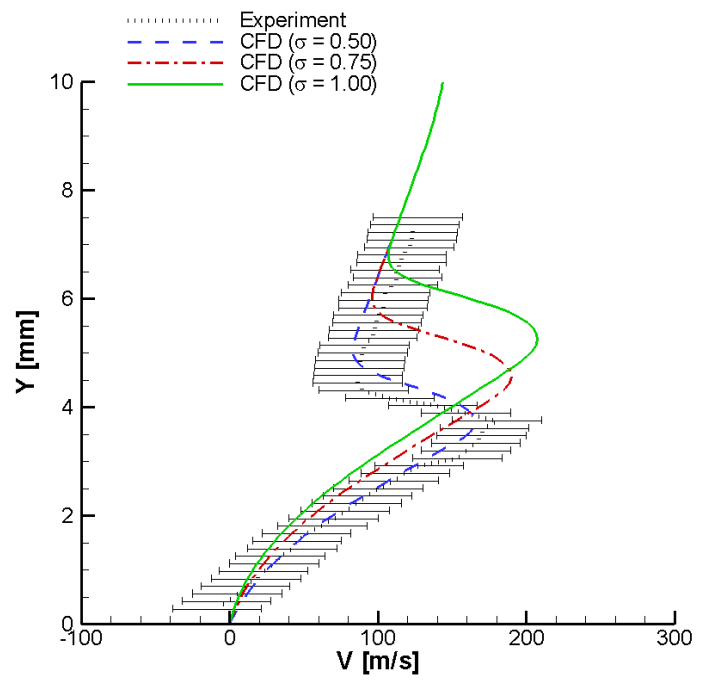

(e) $\mathrm{x}=12.5 \mathrm{~mm}$

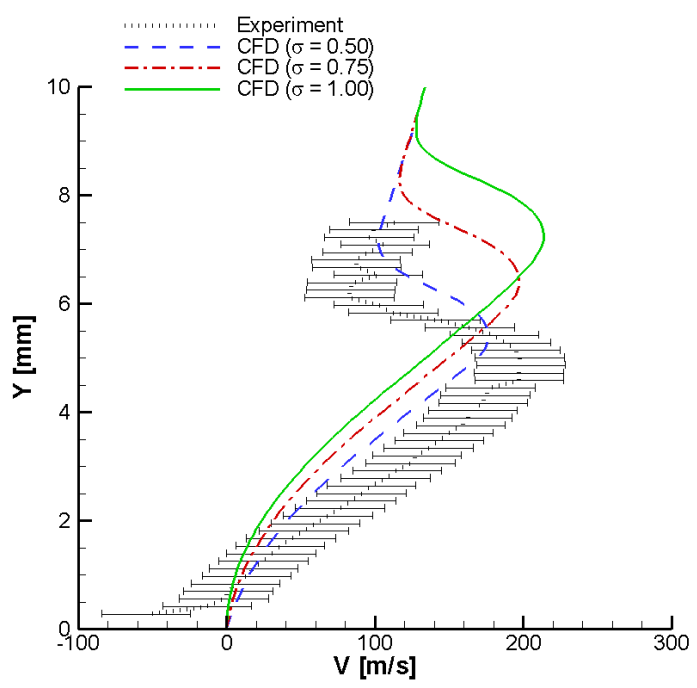

(g) $\mathrm{x}=17.5 \mathrm{~mm}$

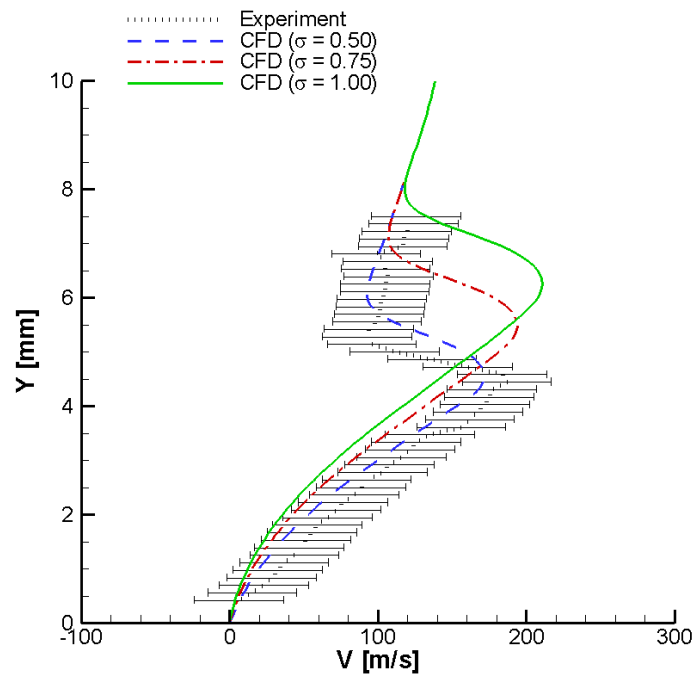

(f) $\mathrm{x}=15.0 \mathrm{~mm}$

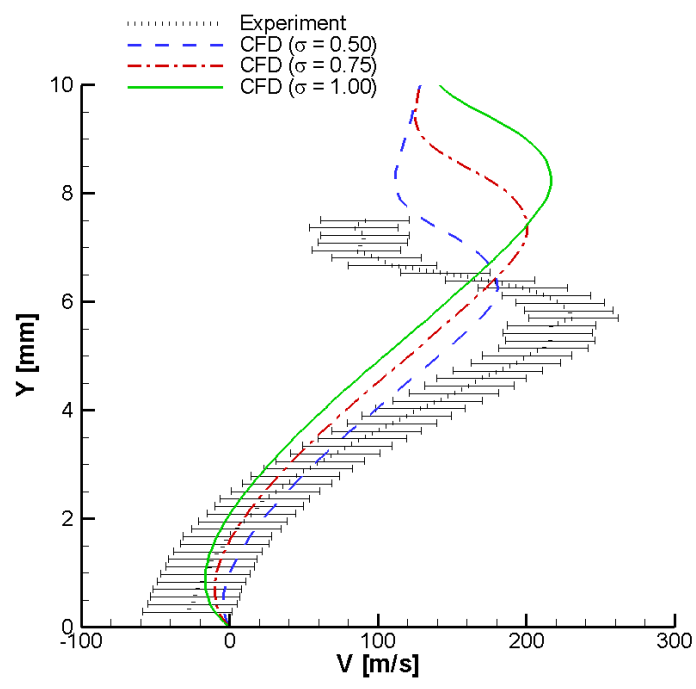

(h) $\mathrm{x}=20.0 \mathrm{~mm}$

Figure 8. Velocity component normal to the surface (V) for a hypersonic flow over a flat plate (cont.). The computed velocity profiles through the shock match best with experiment for lower values of surface accommodation. Simulation results do not agree well with the experimental values near the trailing edge due to an adverse pressure gradient in the experimental results. 
for the wedge that CFD tends to underpredict the value of the velocity slip near the leading edge.

The previous DSMC results ${ }^{11}$ also showed that simulations employing lower values of the accommodation coefficient tended to agree better for the $V$-velocity. However, the DSMC results agreed best for higher levels of surface accommodation $\left(\sigma_{D S M C}=1.0\right.$ rather than $\sigma_{C F D}=70$ for $U$, and $\sigma_{D S M C}=0.75$ rather than $\sigma_{C F D}=0.5$ for $V)$.

\section{Surface Properties}

Although there are no experimental results for surface pressure and shear stress, it is instructive to compare the numerical results at the different values of accommodation coefficient. Figure 9 compares the surface pressure and shear stress along the flat plate. The profiles are very similar to those for the wedge; a large peak at the leading edge is gradually reduced along the surface. As expected, lower levels of surface accommodation reduce the shear stress on the surface. The surface pressure is also reduced with lower values of surface accommodation as the pressure on a flat plate is due solely to viscous effects.

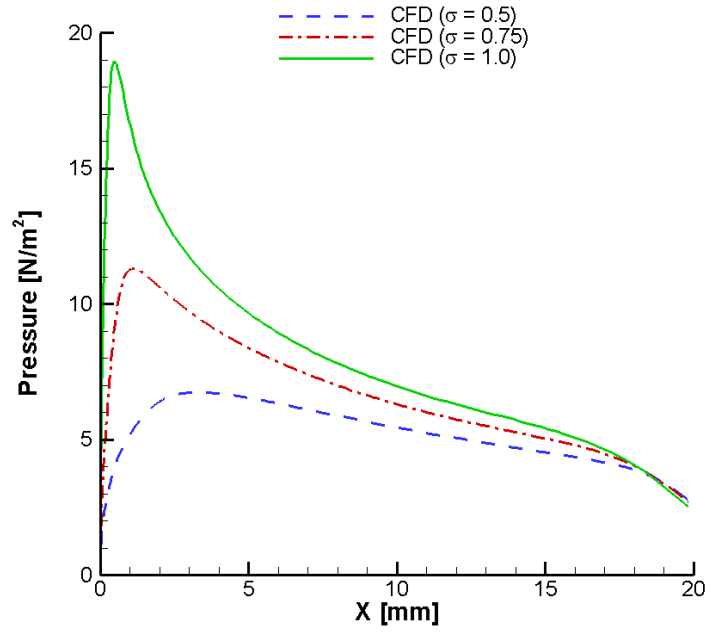

(a) Pressure

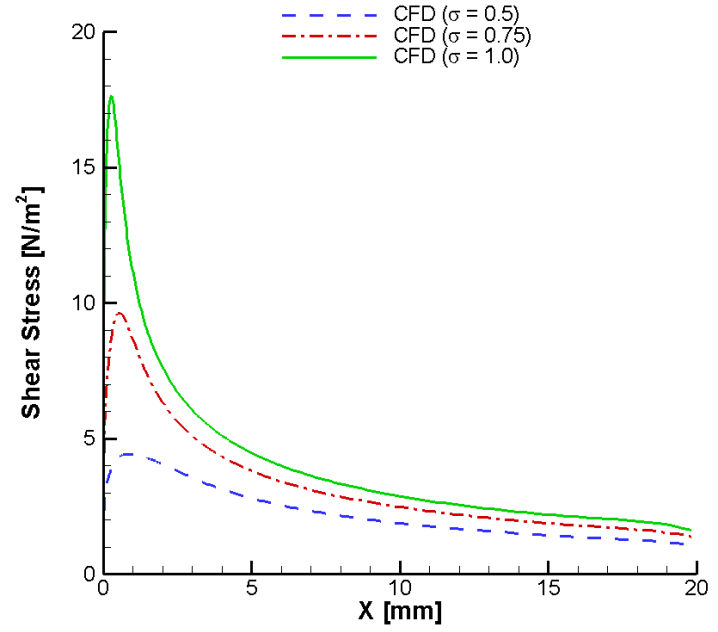

(b) Shear stress

Figure 9. Surface pressure and shear stress for a hypersonic flow over a flat plate. Decreasing the surface accommodation decreases the viscous effects, decreasing the pressure and shear stress.

The experimental values are extrapolated to the surface and plotted with the computational results for velocity slip in Figure 10. As the flow progresses along the plate the large value at the leading edge is reduced to an almost constant value. The large reduction in experimental velocity slip near the trailing edge is also due to the adverse pressure gradient. Extrapolating the experimental velocity slip from the $U$ profiles show that near the middle of the plate, at about $x=10 \mathrm{~mm}$, the experimental values fall between the flow solutions with $\sigma=0.75$ and $\sigma=0.5$.

\section{Summary}

CFD simulations of a hypersonic flow over a flat plate are compared with experimental results and previously obtained DSMC results. Although there is some evidence of flow separation near the trailing edge of the plate in the experimental results, CFD velocity data agrees fairly well with the experimental data at other locations along the plate. For the velocity component parallel to the flow $(U)$, results obtained using a surface accommodation coefficient value of 0.5 agrees better with the experimental results near the leading edge, while further along the plate an accommodation coefficient value between 0.75 and 1.0 provides best agreement. The lower accommodation value near the leading edge effectively increases the slip velocity; CFD also under-predicted the velocity slip near the leading edge of the wedge. For the wall normal velocity component $(V)$, an accommodation coefficient of 0.5 gives best agreement with experimental data. Previous DSMC simulations showed best agreement with the experimental data for higher values of the accommodation coefficient. 


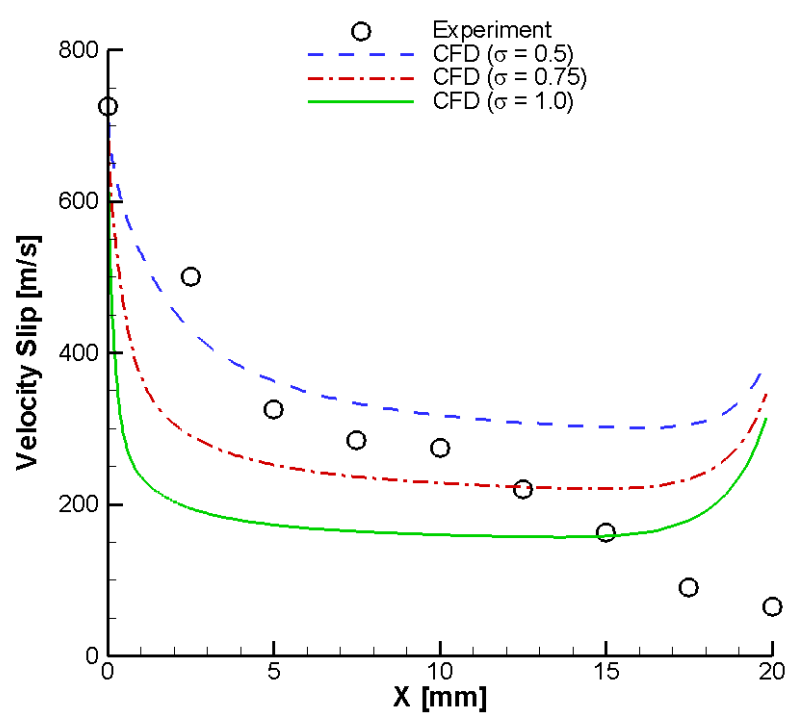

Figure 10. Velocity slip for a hypersonic flow over a flat plate. Reduction in surface accommodation increases velocity slip. The large decrease in the experimental velocity slip near the trailing edge is most likely due to an adverse pressure gradient.

Less surface accommodation significantly decreases the predicted pressure and shear stress near the leading edge.

\section{Acknowledgments}

This work was sponsored in part by the Space Vehicle Technology Institute, under NASA grant NCC3-989 with joint sponsorship from the U.S. Department of Defense and the U.S. Air Force Office of Scientific Research through grant FA9550-05-1-0115.

\section{References}

${ }^{1}$ Bird, G. A. Gas Dynamics and the Direct Simulation of Gas Flows. Oxford University Press, Oxford, 1994.

${ }^{2}$ Blottner, F. G., Johnson, M., And Eluis, M. Chemically reacting viscous flow program for multi-component gas mixtures. Tech. Rep. SC-RR-70-754, Sandia Laboratories, Albuquerque, New Mexico, 1971.

${ }^{3}$ Boyd, I. D., Chen, G., And Candler, G. V. Predicting failure of the continuum fluid equations in transitional hypersonic flows. Physics of Fluids 7, 1 (Jan. 1995), 210-219.

${ }^{4}$ Cecil, E., And McDaniel, J. C. Planar velocity and temperature measurements in rarefied hypersonic flow using iodine LIF. AIAA Paper 2005-4695.

${ }^{5}$ GöкÇEn, T., AND MacCoRmack, R. W. Nonequilibrium effects for hypersonic transitional flows using continuum approach. AIAA Paper $1989-0461$

${ }^{6}$ Lockerby, D. A., Reese, J. M., AND Gallis, M. A. Capturing the Knudsen layer in continuum-fluid models of nonequilibrium gas flows. AIAA Journal 43, 6 (June 2005), 1391-1393.

${ }^{7}$ Lofthouse, A. J., AND Boyd, I. D. Nonequilibrium aerothermodynamics of sharp leading edges. AIAA Paper 2009-1316.

${ }^{8}$ Lofthouse, A. J., Boyd, I. D., AND Wright, M. J. Effects of continuum breakdown on hypersonic aerothermodynamics. Physics of Fluids 19, 2 (2007), 027105.

${ }^{9}$ Lofthouse, A. J., Scalabrin, L. C., AND Boyd, I. D. Hypersonic aerothermodynamics analysis across nonequilibrium regimes using continuum and particle methods. AIAA Paper 2007-3903.

${ }^{10}$ Lofthouse, A. J., Scalabrin, L. C., AND Boyd, I. D. Velocity slip and temperature jump in hypersonic aerothermodynamics. Journal of Thermophysics and Heat Transfer 22, 1 (2008), 38-49.

${ }^{11}$ Padilla, J. S., AND Boyd, I. D. Assessment of gas-surface interaction models in DSMC analysis of rarefied hypersonic flow. AIAA Paper 2007-3891.

${ }^{12}$ Schwartzentruber, T. E., Scalabrin, L. C., and Boyd, I. D. A modular particle-continuum numerical method for hypersonic nonequilibrium gas flows. Journal of Computational Physics 225 (July 2007), 1159-1174. 
${ }^{13}$ WAng, W.-L. A Hybrid Particle/Continuum Approach for Nonequilibrium Hypersonic Flows. PhD thesis, The University of Michigan, Ann Arbor, MI, 2004. 